\title{
ACTIVITY AND ADVANCED CANCER: A GROUNDED THEORY
}

Sonya S. Lowe, M.D. Ph.D. (Corresponding Author)

Department of Symptom Control and Palliative Care

Room 2001, Cross Cancer Institute

11560 University Avenue NW

Edmonton, Alberta, Canada T6G 1 Z2

Email: Sonya.Lowe@ahs.ca

\author{
Christine Milligan, Ph.D. \\ Professor, Division of Health Research \\ Faculty of Health \& Medicine, Lancaster University \\ Lancaster, Lancashire, United Kingdom LA1 4YG \\ Email: c.milligan@lancaster.ac.uk
}

Sarah G. Brearley, Ph.D.

Senior Lecturer, Division of Health Research

Faculty of Health \& Medicine, Lancaster University

Lancaster, Lancashire, United Kingdom LA1 4YG

Email: sarah.brearley@lancaster.ac.uk 


\section{Funding: Alberta Cancer Foundation}

Date: August 20, 2019

Keywords: grounded theory, exercise, palliative care, neoplasms, quality of life 


\section{Abstract}

Background: Cancer-related fatigue and loss of physical functioning are distressing symptoms which negatively impact the quality of life of people with advanced cancer. Physical activity has been shown to have positive effects on these symptoms in early stage cancer, but previous research demonstrated an incongruence between people with advanced cancer's expressed interest and actual participation in a physical activity intervention. Aim: To gain an in-depth understanding of the experience of activity and quality of life in people with advanced cancer, using a classic grounded theory approach. Design: Through the post-positivist lens of subtle realism, and informed by classic grounded theory methods, a two-phase, cross-sectional, qualitative study was conducted. For seven days duration, participants wore an activPAL ${ }^{\mathrm{TM}}$ activity monitor and completed a daily record sheet, which were then used as qualitative probes for face-to-face, semi-structured interviews. Setting/Participants: A total of 15 people with advanced cancer, aged 18 years or older, and with a median survival of 100 days from time of study consent, were recruited from an outpatient department of a tertiary cancer centre in Alberta, Canada. Findings: Maintaining their responsibilities, no matter how small, was the prime motive for participants' behaviour. For people with advanced cancer, the minimum level of responsibility was dynamic and unique. It was achieved through a multifaceted interaction between the perceived benefits, prevailing conditions and mechanisms. Conclusions: This grounded theory enables understanding of activity as a mechanism through which responsibility is managed, and may inform future behavioural interventions in people with advanced cancer. 


\section{What is already known about the topic}

- Cancer-related fatigue and loss of physical functioning contribute to poor quality of life in people with advanced cancer.

- Physical activity improves cancer-related fatigue and physical functioning outcomes in people with early stage cancer.

- Previous research has shown a discrepancy between people with advanced cancer's expressed interest and actual participation in physical activity.

\section{What this paper adds}

- For people with advanced cancer, the primary driver underlying their day-to-day behaviour was to maintain their responsibilities.

- Activity was meaningful to people with advanced cancer insofar as it enabled them to meet their minimum level of responsibility.

- People with advanced cancer perceived activity as one of a number of mechanisms for maintaining their responsibilities.

\section{Implications for practice, theory or policy}

- This is the first integrated theoretical framework which explains the conceptual relationships between maintaining their responsibilities and the day-to-day behaviour of people with advanced cancer.

- The development of future behavioural interventions should be focused on the minimum level of responsibility that is uniquely defined by each person with advanced cancer. 
- Interdisciplinary team members should pay close attention to the underlying reasons for individual patterns of behaviour in people with advanced cancer. 


\section{Introduction}

Cancer-related fatigue and loss of physical functioning are amongst the most distressing symptoms experienced by cancer patients, with detrimental impact on their quality of life..$^{3,4}$ Physical activity interventions have been shown to improve fatigue and physical functioning outcomes in people with early stage cancer. ${ }^{5-7}$ Given that cancer progression is characterized by worsened fatigue, loss of physical functioning and decline in overall quality of life, ${ }^{8}$ there is increasing attention on whether physical activity can positively impact these outcomes in people with advanced cancer. A systematic review investigating the safety and feasibility of exercise interventions in patients with advanced cancer concluded that exercise interventions appear to be safe and feasible. ${ }^{9}$ It is unclear, however, when during the cancer trajectory these findings would continue to apply.

A pilot survey of 50 participants with advanced cancer found that the majority indicated they would be interested in and felt able to participate in a physical activity intervention. ${ }^{10} \mathrm{~A}$ homebased functional walking program was developed for a feasibility trial, informed by the participants' interests and preferences. ${ }^{11}$ Low recruitment and high attrition (only three of the nine recruited participants completing the intervention) demonstrated an incongruence between the high level of interest in physical activity expressed by people with advanced cancer, ${ }^{10,11}$ and their low level of actual participation in a physical activity intervention. ${ }^{12}$

This study aimed to gain an in-depth understanding of the experience of activity and quality of life in people with advanced cancer. The objectives were: 1) to explore the meaning of activity for people with advanced cancer in the context of their day-to-day life, 2) to elicit people with 
advanced cancer's perceptions of activity with respect to their quality of life, and 3) to elicit people with advanced cancer' views of barriers and facilitators to activity in the context of their day-to-day life.

\section{Methods}

Following ethical approval by the Alberta Health Services Cancer Research Ethics Committee (26163), University of Alberta Health Research Ethics Board (26163), and Lancaster University Faculty of Health and Medicine Research Ethics Committee, a two-phase, cross-sectional study was undertaken using classic grounded theory approach. ${ }^{13,14}$ This study was conducted through the post-positivist lens of subtle realism, which endorses activity as an independent and tangible entity, but which can only be accessed indirectly through understanding others' perspectives of that reality. ${ }^{15,16}$ Detailed methods are outlined in the study protocol. ${ }^{17}$

\section{Literature Review}

True to classic grounded theory method, a literature review was not conducted a priori so as not to impose preconceived ideas or assumptions on the emergent theoretical framework. ${ }^{18}$ Because of the previous systematic review in physical activity and advanced cancer, ${ }^{19}$ a true tabula rasa perspective could not be attained, nor do Glaser and Strauss endorse this. ${ }^{13}$ The findings from previous studies, ${ }^{10,12,20}$ demonstrated an incongruence between people with advanced cancer's expressed interest and actual participation in physical activity, and provided the context from which the impetus for this study originated.

\section{Setting}


This study was undertaken at the Department of Symptom Control and Palliative Care, Cross Cancer Institute in Edmonton, Canada. The Cross Cancer Institute serves a catchment area of nearly 1.5 million people, and is one of two tertiary cancer centres in the province of Alberta. ${ }^{21}$

\section{Sample}

People with advanced cancer, aged 18 years or older, and with a clinician-estimated life expectancy of less than 12 months, were recruited. ${ }^{17}$ Interdisciplinary team members screened all outpatient referrals for eligibility criteria (see Box 1), and approached potential participants for their verbal permission to contact them regarding the study. The researcher (SL) was introduced as a PhD student, but her professional background as a physician with training in clinical communication was not disclosed. The researcher had no clinical contact with participants before, during or after the study was conducted. Following verbal permission to be contacted, the participants were telephoned by the researcher and recruited into the study. All potential participants who were approached, agreed to participate in the study. 
Inclusion Criteria

- 18 years of age or older

- $\quad$ Ability to understand, provide written informed consent in, and speak English

- Diagnosis of advanced cancer, which is defined as progressive, incurable, and locally recurrent or metastatic disease

- Clinician-estimated life expectancy of less than 12 months

- Cognitively intact, which is defined as a Mini-Mental State examination score at or above the level expected for person's age and education level ${ }^{1}$

- Approval of attending physician.

Exclusion Criteria

- Palliative Performance Scale level of $30 \%$ or less ${ }^{2}$, and

- Any person who, in the opinion of the treating physician, is within the last days to hours of life

\section{Box 1: Study Eligibility Criteria}

\section{Sampling}

In keeping with classic grounded theory method, an initial cycle of open sampling was guided only by a general understanding on where activity, the phenomenon of interest, resided conceptually. ${ }^{22}$ As concurrent data collection, coding and analysis proceeded, the emerging theory directed which groups or subgroups to turn to next, in the subsequent cycle of theoretical sampling. Theoretical saturation was the criteria by which sampling of different groups that were relevant to the core categories of the emerging theory ceased, and which signified the end of concurrent data collection, coding and analysis. 


\section{Participants}

A total of 15 people with advanced cancer participated in the study, from March 2014 to

January 2015 (see Table 1). No participants withdrew from the study. As of March 2017, all

study participants were deceased, with a median survival of 100 days from date of study entry

to date of death. 


\section{Table 1. Participant Characteristics}

Participants in Open Sampling Cycle $(n=10)$

\begin{tabular}{|c|c|}
\hline Age (years), median (IQR) & $74.5(60.0-79.0)$ \\
\hline Gender (female), n (\%) & $5(50.0)$ \\
\hline \multicolumn{2}{|l|}{ Cancer Diagnosis, n (\%) } \\
\hline Lung & $2(20.0)$ \\
\hline Oesophagus & $1(10.0)$ \\
\hline Kidney & $1(10.0)$ \\
\hline Colon & $1(10.0)$ \\
\hline Melanoma & $1(10.0)$ \\
\hline Lymphoma & $1(10.0)$ \\
\hline Parotid Gland & $1(10.0)$ \\
\hline Peritoneum & $1(10.0)$ \\
\hline Cervix & $1(10.0)$ \\
\hline \multicolumn{2}{|l|}{ Current Treatment } \\
\hline Palliative Chemotherapy & $3(30.0)$ \\
\hline Palliative Radiotherapy & $1(10.0)$ \\
\hline None & $6(60.0)$ \\
\hline \multicolumn{2}{|l|}{ Caregiver Present, n (\%) } \\
\hline Spouse & $6(60.0)$ \\
\hline Child & $1(10.0)$ \\
\hline None & $3(10.0)$ \\
\hline Survival from Date of Study Entry, median (IQR) & $113.5(59.5-270.8)$ \\
\hline \multicolumn{2}{|l|}{ Participants in Theoretical Sampling Cycle $(n=5)$} \\
\hline Age (years), median (IQR) & $53(38.0-56.5)$ \\
\hline Gender (female), n (\%) & $4(80.0)$ \\
\hline \multicolumn{2}{|l|}{ Cancer Diagnosis, $\mathrm{n}(\%)$} \\
\hline Breast & $2(40.0)$ \\
\hline Colon & $1(20.0)$ \\
\hline Pancreas & $1(20.0)$ \\
\hline Cervix & $1(20.0)$ \\
\hline Cervix & $1(20.0)$ \\
\hline \multicolumn{2}{|l|}{ Current Treatment } \\
\hline Palliative Chemotherapy & $2(40.0)$ \\
\hline Palliative Radiotherapy & $1(20.0)$ \\
\hline None & $2(40.0)$ \\
\hline \multicolumn{2}{|l|}{ Caregiver Present, n (\%) } \\
\hline Spouse / Partner & $2(40.0)$ \\
\hline Parent & $1(20.0)$ \\
\hline None & $2(40.0)$ \\
\hline Survival from Date of Study Entry, median (IQR) & $94.0(52.0-128.0)$ \\
\hline
\end{tabular}




\section{Data Collection}

The study consisted of two phases of data collection. During Phase One (seven days duration), participants wore an activPAL ${ }^{\mathrm{TM}}$ activity monitor (PAL Technologies Ltd, Glasgow, UK) and completed a daily record sheet. ${ }^{17}$ During Phase Two, face-to-face, semi-structured interviews were conducted by the researcher (SL) with each participant. In conjunction with the semistructured interview guide (see Appendix A), the activity monitor output and daily record sheets were used as qualitative probes, informing and individualizing interview questions to glean insight into what was relevant and important from the participant's perspective. ${ }^{23}$ Prior to the onset of Phase One, the researcher defined activity for the participants as any bodily movement produced by the skeletal muscles that results in a substantial increase in energy expenditure over resting levels. ${ }^{24}$ The activity monitor output from Phase One was subsequently printed and shared with the participants during Phase Two, and formed the basis of semi-structured interview questions exploring their perceptions and meaning of activity (see Appendix A).

Data collection for Phase Two took place in a location of the participants' choosing (home $(n=9)$ and tertiary cancer centre $(n=6))$. Caregivers could be present but their views were not specifically elicited. Each participant was interviewed once, with no repeat interviews. The median interview duration was 59 minutes (range 39-96 minutes). All interviews was recorded using a digital audio recorder, and first impressions and observations were recorded as field notes during and immediately after each interview. The researcher transcribed the digital audio recordings after conducting each interview. All data were uploaded onto ATLAS.ti ${ }^{\mathrm{TM}}$ qualitative data analysis software to facilitate application of codes and memos across interview transcripts 
and activity monitor outputs. ${ }^{25}$ Participant feedback was not solicited for either interview transcripts or findings due to the limited life expectancy of participants.

\section{Analysis}

As per classic grounded theory methods, ${ }^{22}$ line-by-line, open codification on each interview transcript was performed by the researcher (SL), with integrated reference to interview field notes, the participant's activity monitor output and their daily symptom record. Preliminary codes were developed to group data together and encapsulate the main concepts emerging from the data. Constant comparative analysis was employed in order to generate conceptual categories, properties and hypotheses that were directly relevant to the emerging core category. ${ }^{26}$ Selective coding followed wherein only those factors that related to the emerging core category were analysed. ${ }^{22}$ Theoretical memos were used throughout coding and analysis, and were printed and sorted by hand to facilitate emergence of conceptual categories, properties and dimensions, and ultimate integration of the theory. ${ }^{27}$

\section{Core Conceptual Category}

A core conceptual category distinguishes itself by the following characteristics: 1) it is central in its relationship to other categories and their properties, and 2) it explains a large degree of variation in the behaviour of participants. ${ }^{22}$ Through constant comparison of incidents within the data, the critical threshold of responsibility emerged as a potential core category in September 2014, and appeared to encompass the multivariate day-to-day experiences of participants as revealed through their interviews, as well as the diversity in physical activity patterns as identified through activPAL ${ }^{\mathrm{TM}}$ monitoring. Thus the critical threshold became the 
core conceptual category from which further theoretical sampling ensued, starting in November 2014. The ensuing transition from open to selective coding was then focused upon developing the properties and dimensions of the sub-categories of the critical threshold, and subsequent codes delimited to 42 unique code families. Through constant comparative analysis, theoretical sampling and memoing, the core category of the critical threshold emerged as a typology. This is congruent with Glaser's contention that basic social processes are merely one type of core category, but not all core categories are basic social processes. ${ }^{27}$

\section{Theoretical Saturation}

Concurrent coding and constant comparative analysis revealed that: a) the range of data were maximized relevant to the emergent core category of the Critical Threshold, b) the initial gaps within the core category were filled, and c) there was recurrence of similar instances with no other additional data contributing to the development of the properties of the Critical Threshold. Thus theoretical saturation was achieved in keeping with a classic grounded theory approach.

\section{Results}

\section{Maintaining Responsibility: The Participant's Main Concern}

In this study of people with advanced cancer, Maintaining Responsibility was the main issue that occupied much of their activity and behaviour. ${ }^{14}$ Responsibilities were viewed as duties or tasks which were incumbent upon the participant to fulfil. These responsibilities were specific to each person with advanced cancer, and their understanding of their disease and its prognosis impacted the choice about which particular obligations to devote their limited time and energy 
to. The participants' focus was not on being more active, but rather what activity enabled them to do.

ACT14: [pause] That's life, that's part of life. It's, it's the life code, it's [pause] you have family, you help your family. You open up a business, it's your responsibility to keep it running. If you have pets, they're your responsibilities to take over. If you have children, it's your responsibility to raise them properly... I'd love to be active enough to participate in that, the next 3-6 months, to get my ducks in a row... I've got to speed up to slow down.

\section{Critical Threshold: The Core Conceptual Category}

The Critical Threshold emerged as the core conceptual category; it is the minimum level of responsibility to be met at a point in time, uniquely defined by each participant. The ability of participants to do even small tasks was challenged by disease progression and symptom burden, and thus the Critical Threshold changed, particularly towards the end of life. Even when a task cannot be completed independently, there can still be ways to feasibly meet the minimum level of responsibility. People with advanced cancer perceived the aspects required to meet the Critical Threshold as necessary.

Three distinct sub-categories of the Critical Threshold emerged: Provision for Self, Key Relations and Commitment to Community (see Table 2). With respect to Provision for Self, the person with advanced cancer can address each of its three dimensions (self-care, self-management of health and self-sufficiency) in order to meet the Critical Threshold. With respect to Key Relations, the person with advanced cancer can address one or more of its five dimensions (role 
as caregiver, role as spouse, role as parent, role as adult child, and role as grandparent) in order to meet the Critical Threshold. With respect to Commitment to Community, the person with advanced cancer can address one or both of its dimensions (vocation and society) in order to meet the Critical Threshold. 
Table 2. The Critical Threshold: Sub-Categories and Dimensions

TYPOLOGY MEANING FOR PARTICIPANTS

ILLUSTRATIVE QUOTATION

CORE CONCEPTUAL CATEGORY

Critical Threshold

The minimum level of responsibility to be met

at that point in time

"It seems to me like I was going like this [motioning a downward trajectory], like physically I was going like this, but I maintained my activity level. I don't think I would have done any necessarily more or less...but in general I just did what needed to be done, right?" (ACT15)

\section{SUB-CATEGORY}

Provision for Self

Doing what is needed to exist

\section{"Yeah I think right now I feel like I'm just} surviving, you're not really living, not doing things, you're just surviving, that's it." (ACT10)

\section{DIMENSION}

Self-Care

Self-Management of Health
Being accountable and advocating for personal well-being
"But sometimes my body just says sleep, and I do it, at this stage, stage 4 , I think you need to listen to that too, and not worry about the time of day or anything, you know." (АСT09)

"I'm just trying to look after myself as best as I can, and then not getting too tired and uh you know one of the first things they said...was you know, not to have stress in 
your life, and that's what I'm trying to do" (ACT04)

Self-Sufficiency

Self-Sufficiency

cy

Being able to manage independently, without being a burden on others "being able to fend for myself as much as possible...I mean I realize I can't do everything anymore, and that's hard to take, but I'm getting used to that." (АСT09)

\section{SUB-CATEGORY}

Key Relations
Obligations which participants assume within their households and families
"Spending time with my family has always been a high priority for me. It's always been that way. But now I'm doing my best to make sure everybody has time... whereas it wasn't so important before, right? But now I'm making sure that everybody has time, yeah." (ACT11)

\section{DIMENSION}

Role of Caregiver

Role of Spouse
Responsibility for the welfare of dependents

Responsibility towards partner

\section{"Because if I went to bed around that time, I} take the dog out every 4.5 hours or so, so that would be taking him out again, just to the balcony, he's too small to go [outside by himself], yeah, and for me it's hard to get up and down the stairs, so...it's getting better, but, yeah he's too tiny to, he just goes on the balcony. You saw him." (ACT10)

"I would like to [do more activity], but uh, [her spouse] has had a couple of bouts with you know, sort of, passing out or small TIA or something, and I don't like to leave him 


\section{Role of Parent}

Role of Adult Child

Role of Grandparent
Responsibility towards children

Responsibility towards parents

Responsibility towards grandchildren alone, so if I'm going out, then I want to have somebody here with him." (АСTO4)

" [The end is] coming quicker than we thought, but that's all I can really do is sort of reassure them, that life will go on for them, and it is as it is. That's basically all I can offer I guess at this point, I think. It's not a lot of reassurance or anything, but I think it's what I can offer them as their parent." (ACT06)

"[My mother] is [dependent on me], but I've worked hard at getting that so it's not that way. I don't want her to [pause] have too hard a time when I go...she spends time with me without expecting me to spend time with her, so it's better that way." (ACT11)

"at one point we were walking in the yard, and she was holding my hand, and she looked at me and she says "Nanny", she says, "I know you have to go to heaven" but she said "I don't want you to go today." So...they're working their way through it, yeah [pause]. But it's not something that I'm hiding even from them." (ACT11)

\section{SUB-CATEGORY}

\section{Commitment to Community}

Responsibilities which participants assume, in both formal and informal roles, with regards to fellow individuals
"And I do a lot of volunteer things too, they're also extremely important to me, so. Um, so that's another thing that people are telling 


\section{DIMENSION}

Vocation

Responsibilities which participants assume within their work or occupation

Society
Responsibility for the welfare of others and accountability to the collective good
"I mean that's part of why I got into what I do, so not having that is like double, double whammy almost, number one [I'm] not working...I think that is important because I don't know, I love what I do, I love helping the kids, you know...it's just [pause] it really sucks not being able to do any of that, you know?" (ACT10)

"I'm president of the [local political association], so we're getting ready for election, and then I'm also the chair of the [learning and literacy association], and I have other things too that I have, that are on the back burner, but those two things I keep up with...so then I'm always thinking, well should drop them, or you know, should resign from them, but...[pause] so far I haven't. It's a big thing for me too, community, building community, contributing, yeah, that's a big thing for me, yeah." (ACT15) 
In practice, the degree to which the Critical Threshold encompassed these three sub-categories was unique to each person with advanced cancer, and changed over the course of the disease (see Figure 1). Each subcategory, and each dimension, was independent of the others. The dayto-day activity of people with advanced cancer was focused on one or more of these subcategories, and one of more of their dimensions, in order to meet the Critical Threshold. Participants engaged in activity in order to meet their minimum level of responsibility.

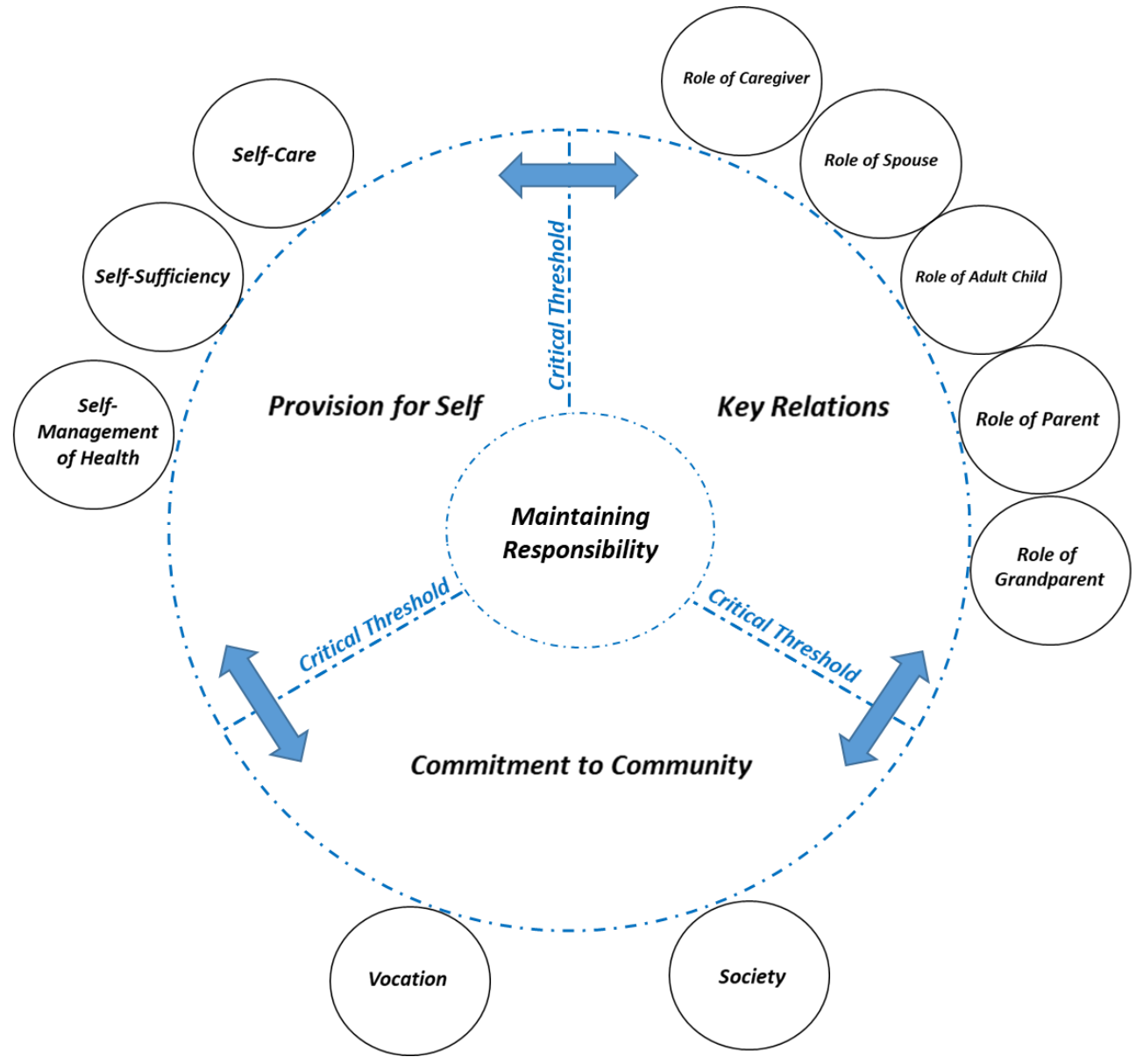

Figure 1: The Critical Threshold: Maintaining the Minimum Level of Responsibility 


\section{Downsizing: Meeting the Critical Threshold}

Downsizing was the decision-making strategy used by participants to scale back to the minimum level of responsibility at any given moment. Downsizing involved participants asking the following questions of themselves: 1 ) what were they still able to do with the time that was remaining, 2) what were the most important responsibilities to address given limited prognosis, and 3) at that point in time, what should they be doing that reflects these priorities. At the end of life, participants expended the minimum energy required to fulfil their obligations, and did no more than that.

Downsizing is influenced by the dynamic interplay between the perceived benefits (purpose, contribution, accomplishment, identity, locus of control) and prevailing conditions (understanding of illness, symptom burden, physical functioning, disease and symptom treatment strategies, day-to-day fluctuations, acute stressors, environmental layout, equipment supports and person supports) at any given moment (see Table 3). The combination of benefits and conditions also influences the choice of mechanisms (delegating, transferring, goal-setting, day-to-day routine, the path of least resistance, pacing, and activity) which enable people with advanced cancer to meet their critical threshold. Activity was perceived by participants as one potential mechanism which enabled them to meet their minimum level of responsibility. 
Table 3. Downsizing to the Critical Threshold: Benefits, Conditions and Mechanisms

TYPOLOGY

CATEGORY

Downsizing
MEANING FOR PARTICIPANTS

Scaling back to the Critical Threshold

\section{ILLUSTRATIVE QUOTATION}

"I'm not sure what's happening with work, I might just say l'm done...it's becoming less important right? I'd really rather just be doing temple and qi gong and you know, so that's kind of what I'm hoping, that I might just move more into that, forget working, we'll see." (ACT15)

\section{SUB-CATEGORY}

Benefits

Positive effects which result from meeting the Critical Threshold

"Well I have to be able to do something, I'm not contributing anything to this world right now, and not to me really. I still have to be useful for something...Not just be here, and fill up space sort of thing. I have to, I have to still be able to feel like I'm contributing." (АСТ03)

DIMENSION

Purpose

The reason for which a certain task or duty is carried out

Contribution

The part the participant plays in carrying out a task or duty

Identity

Reinforcing personal values and beliefs, and regaining a sense of individuality and self
"To me, too, laundry's personal...and that's the way my mother taught me, you know. So I'm particular, so that, and it does give me, it also does give me a sense of purpose, that I'm doing something for myself and I'm not relying on my daughter or my son." (АСТ09)

"I want to be doing stuff, I don't want to be laying around in this couch and sitting around and doing nothing. I want to be busy, I want to do stuff, I want to be a part of life... Well even to our family, right? Just contribute. Be a part of everything again, you know?" (ACT13)

"you're not just sitting there and watch life pass you by, you know, I'm sick, but I'm not an invalid, you know? So...I just, I'm going to participate until the end. So to me this is just...I'm living." (ACT11) 
Accomplishment

Achievement of goals or fulfilment of a task to completion

Locus of Control

Preserving control in the face of inevitable decline

"even though it's not a lot, when you are still able to get from $A$ to $B$, and sometimes you need assistance, but still, you're able to do something, you feel like you've accomplished something, and I think that makes you feel better." (АСT06)

"sometimes my daughter will insist on doing this, and I'll say "[her daughter's name]! Let me do something that I can do!" She'll apologize and she'll say, "ok Mom"'” (АСТ09)

SUB-CATEGORY

Conditions
Contingency factors which impact the Critical Threshold

"I'm fine with what it was, you know, you just, you don't know what to expect, you know, it affects people differently too, right? So, I mean I would have liked to have done more, you know but, expectation wasn't there after being through five previous treatments, you know so I mean it was, it was a progression, it got progressively worse, so um yeah, by the end the expectation was that I'm not doing anything this week, so." (ACTO8)

\section{DIMENSION}

Understanding of Illness

Symptom Burden
Knowledge of the progressive, incurable nature of disease and prognostic implications
Intensity and frequency of physical and psychosocial spiritual issues experienced by the participant
“I don't anticipate doing anything that's, that's mindaltering at this point, or physically going to do anything great, so yeah, it's um kind of coming to the end of the road, and recognizing that that's where I am" (АСT06)

"they're very tender and tight, it's just you know I bend them and you can just feel how tight they are, how tight they are and full of fluid, but it's when I have to lift, like even going out to our patio or to our deck, it's a tiny little bit of a step, and it's just like oh! Lifting heavy weights" (АСТ09) 
Physical

\section{Functioning}

Disease and Symptom

\section{Treatment}

Day-to-Day

Fluctuations

Acute Stressors

Events which are unexpected setbacks

Environment

Layout
Perception of actual ability to perform tasks

Interventions directed toward treating the participant's symptoms and disease

Changes in onset, frequency and intensity of symptoms

The physical setting in which the majority of day-to-day activities take place "you know like the other day I was trying to tie a bag onto my balcony to put bottles in, I couldn't even tie the bag because I can't bend, you know. To put, change the garbage bag in my garbage, well you have to bend to do that. To take your dishes out of your dishwasher, you know, you don't think about that stuff until you can't do it." (ACT10)

"I'm on the hormone treatment, its slowing the testosterone down. There's nothing I can do about it other than deal with it...You have to accept it the way it is. [pause] Not like I'm gonna run 100 yards dash down the track so I don't even think about it. [pause]" (ACT01)

"it's most annoying because I feel really good and then all of a sudden, I think I'm going to do something and all of a sudden the dizziness is back again, so yeah." (АСT03)

"It was good knowing like the results, there was that little bit of relief just in knowing, but it wasn't good news, so, yeah, I was really upset obviously, frustrated, um...[pause]...yeah I don't know I didn't feel like doing much basically. Kind of just yeah, I get really like down and then I just don't do anything, I won't make food or eat or anything" (ACT10)

"the only reason we moved was because of my health issues, it wasn't a good house for me...it was three levels and it's on a hill which is so treacherous. wouldn't have been able to get out of the house at all, off the hill." (ACT15) 
Person Supports

\section{Equipment \\ Supports \\ Availability and access to physical aids for activities of daily living}

SUB-CATEGORY

Mechanisms

The working means by which the participant carries out their tasks and obligations.

"Because I have somebody there, right? Like we'll go grocery shopping and stuff and you know he'll come with me, as long as I'm leaning on a cart or something, I'm ok. But still, like he's gotta come with me and helps me out, right? If something's low, he can grab it, stuff like that." (ACT10)

"If there's a wheelchair at the shop, if there's a cart at the shop, if there's some shopping cart that I can push and steady myself on. But mostly, yeah, the walker's mainly for the home." (ACT14)

"I do that quite often, though, I just mentally think of ok, what I have to do, ok and what I can do, that's a little different thing, and then [her friend] is coming to stay with me for the last couple of weeks...and there's a few things I want to do which I don't want to start myself, because I couldn't finish it. So therefore I wait until she comes down." (АСТ03)

DIMENSION Delegating

Temporary assignment of obligations to others, with the expectation that the participant will be able to resume after a defined period of time

Permanent assignment of obligations to others, with little or no expectation that the participant will be able to resume

\section{Transferring}

"I used to do all the grocery shopping and driving here there and everywhere, going to the library, getting books, well, now the gal that cooks, gets the groceries, because I just can't walk around that much." (АCT04) "my daughter, obviously, so I have to make sure, but you know she's really very independent, she's taken on so much, I don't, she knows that I can't do, I can't drive her places as much, and you know she knows that she has to take on extra responsibilities herself too, like I can't shovel, I can't take the dogs out, there's lots of things I can't do. So she knows she has to do them. And she knows that I would be doing them if I could, yeah...but she takes care of them." (ACT15) 
Day-to-Day Routine The participant's typical daily pattern of behaviour, whose structure reflects the Critical Threshold

\section{Path of Least}

Resistance

Pacing

Activity
Minimum energy expenditure required to meet the Critical Threshold

The day-to-day behaviours which enable participants to meet the Critical Threshold
"Oh, my first thing on my list is I'd love to get myself active enough that I can be walking around my home, and getting everything in shape for the next step of my life. I'd love to be... active enough to participate in that, the next 3 to 6 months, to get my ducks in a row." (ACT14)

"usually I find like I wake up, I go lay down for a bit on the couch, and then after that is kind of when I'm the most active like, that's when I have the most, I don't know I seem to be in the least amount of pain, as the day progresses, I get more and more kind of sick, so when I have the energy, I use it to my full advantage, so I'll do the laundry or if there's cleaning that needs to be done, I'll do it then, so. So that kind of time is usually the best for me." (ACT10)

"I feel like maybe I could move around a little bit more, but sometimes it's hard you know you just don't want to, you know, and it goes so easy doing the same thing every day, you just fall into a routine of doing nothing almost" (ACT10)

"maybe like some of the busy days were a little too much, yeah. It burns up my energy...I think if I do like have a busy afternoon, then after I need to rest for awhile, yeah." (ACT12)

"Being active, being able to do the things that I'd like to do, I'd like to be able to do that when I want to do it, but not doing it does not make me feel like I'm missing anything, yeah." (АCT07) 
The degree to which downsizing changes the critical threshold varies depending upon the push and pull of a combination of benefits and conditions; this subsequently influences the mechanisms used for carrying out these responsibilities (see Figure 2). For example, supplemental oxygen can alleviate shortness of breath, thereby enabling the participant to meet the critical threshold. Potential treatment-related sequelae, such as increased fatigue and fatigue, can hinder the participant's ability to meet the critical threshold. Impaired concentration and cognitive sequelae can occur post-palliative chemotherapy, and are barriers to meeting the dimensions of self-sufficiency and accountabilities to vocation. Severe energy depletion can occur post-palliative abdominal paracentesis, and obstructs the participant's ability to meet the sub-category of self-care. 


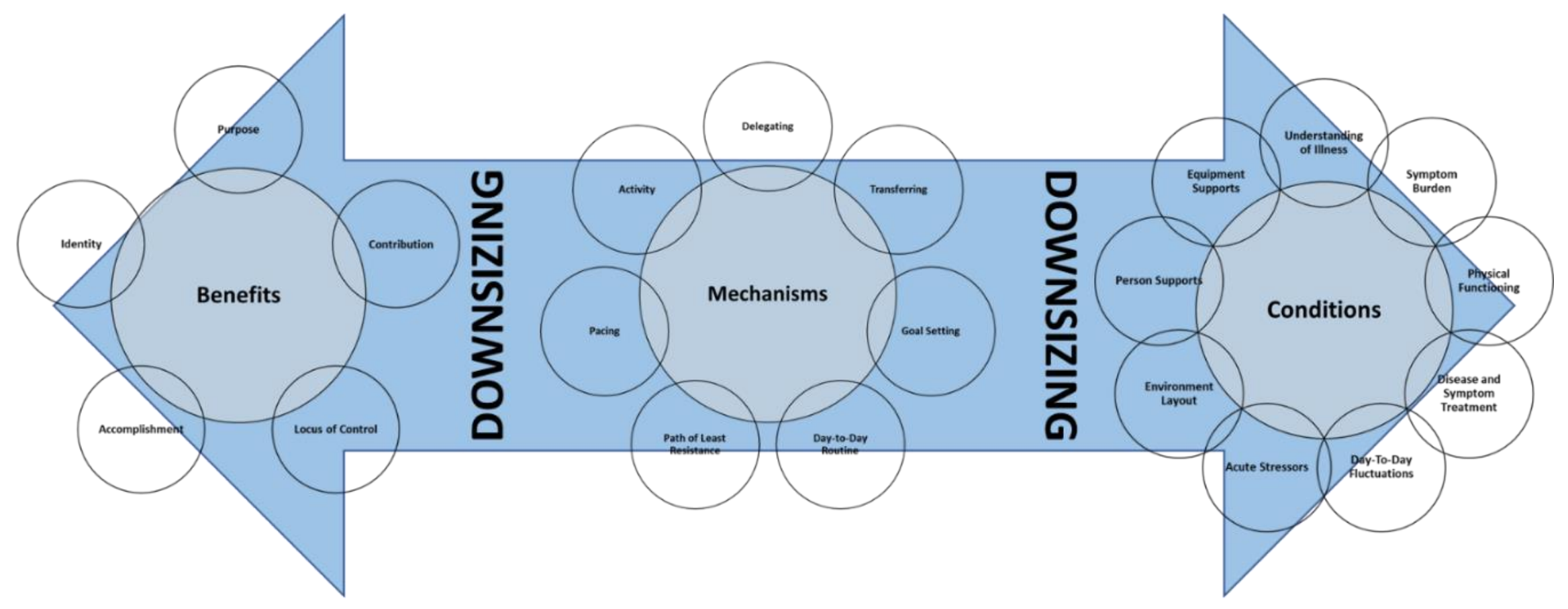

Figure 2: Downsizing: The Push-Pull between Benefits, Conditions and Mechanisms 


\section{The Emergent Theoretical Framework}

Maintaining responsibility, no matter how small, was the main concern which explained the activity and behaviour of people with advanced cancer in this study (see Figure 3). The Critical Threshold was the minimum level of responsibility that was unique to each participant, and emerged as the core conceptual category of this study. For some, the minimum level of responsibility entailed just doing what was needed in order to exist; this could include the obligation to care for themselves, to advocate for their personal well-being, and to be able to manage independently. For others, the minimum level of responsibility could entail personal obligations, such as those associated with family and household roles, or community obligations, such as those attributed to vocation or to society. Downsizing involved making decisions, both consciously and subconsciously, that prioritized the most essential responsibilities. Downsizing resulted in the Critical Threshold being in a state of flux at any given moment, and was influenced by the dynamic interplay between the perceived benefits, prevailing conditions and mechanisms.

People with advanced cancer view activity as the day-to-day behaviours which enable them to meet their critical threshold. Everyday activities, such as bathing and showering, dressing, and functional mobility, enable the person with advanced cancer to meet self-care and provision for self. Other activities such as housework, shopping and transportation, enable the person with advanced cancer to meet self-sufficiency and self-management of health. Activity is prioritized more as a mechanism for meeting the critical threshold, rather than for its own sake. 
The ability to engage in activity, however, is limited by varying conditions at any given moment in time. Prevailing conditions of treatment-related sequelae, high symptom burden, and decline in physical functioning can preclude activity as a mechanism to meet the critical threshold. If the person with advanced cancer experiences nerve-related pain, activity could be limited so as not to aggravate symptom burden. Increased fatigue can be a delayed sequelae of activity, and could be perceived as an additional physical burden. People with advanced cancer could perceive everyday activity as having a high degree of perceived exertion. Depending upon prevailing conditions, activity may not be the preferred mechanism of meeting the Critical Threshold.

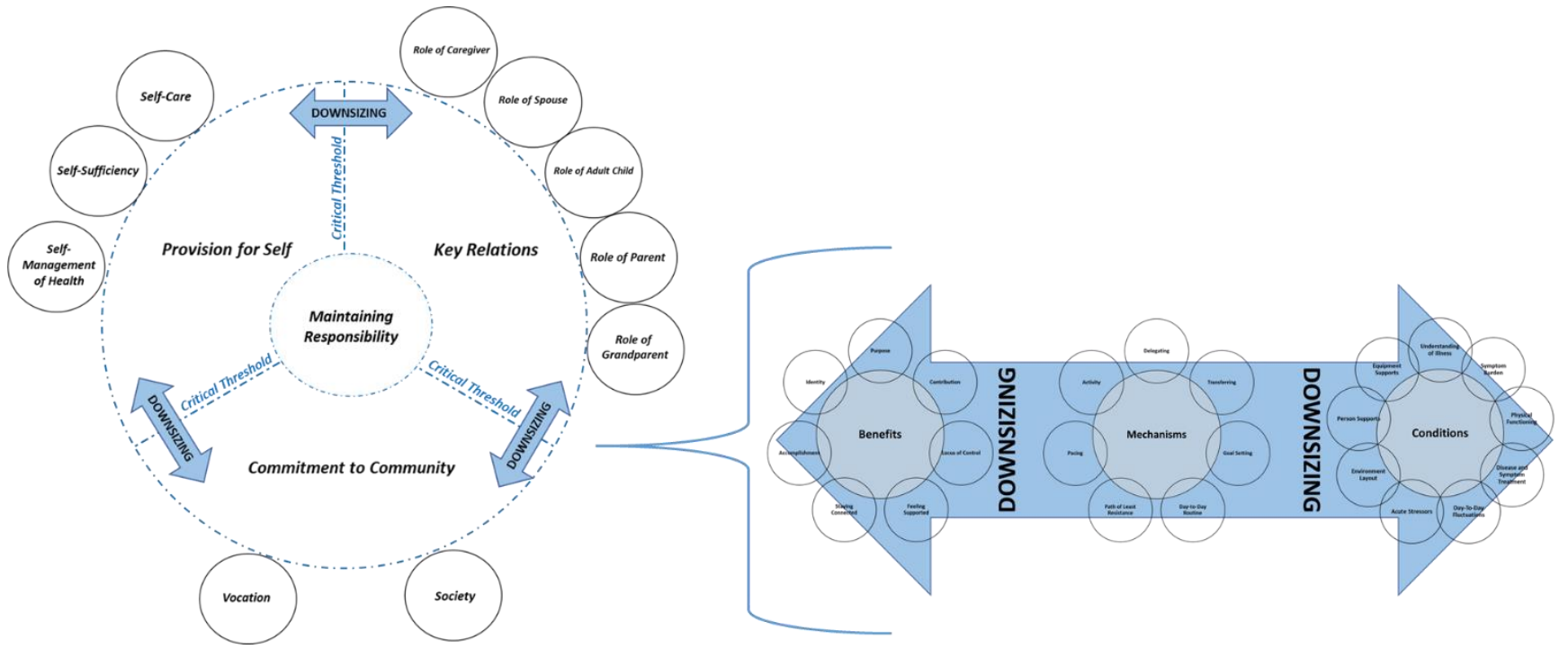

Figure 3: The Emergent Theoretical Framework 


\section{Discussion}

The aim of this study was to gain an in-depth understanding of the experience of activity and quality of life by people with advanced cancer, in order to illuminate the disparity between expressed interest and actual participation in a physical activity intervention, from previous research in this population. ${ }^{11,12}$ By following a grounded theory approach, what emerged from the data was a complex typology with the Critical Threshold, or minimum level of responsibility, being the core conceptual category. Meeting the minimum level of responsibility was the primary explanatory driver underlying the participants' activity and behaviour. On a day-to-day basis, participants were focused on what activity enabled them to do, rather than being active for its own sake. Activity as a mechanism for meeting the Critical Threshold was influenced by the push-pull between perceived benefits and prevailing conditions at any moment in time.

Of the few qualitative studies examining the experience of physical activity in similar populations, the majority focus on participants attending professionally-supervised exercise interventions, ${ }^{28-30}$ given the association between higher functional status and longer survival in people with cancer, ${ }^{31}$ interest in physical activity could be matched by the ability to participate in a physical activity intervention. Participants in this study, however, had a median survival of 100 days from time of study entry to time of death; they were not attending a professionallysupervised physical activity programme. Any level of physical exertion may be all that the participant could undertake. The emergent theoretical framework thus accounts for the incongruence between expressed interest and actual ability of people with advanced cancer to engage in physical activity interventions. 


\section{The Meaning of Activity}

The first objective of this study was to explore the meaning of activity for people with advanced cancer in the context of their day-to-day life. The emergent theoretical framework proposes a critical distinction between the meaning of activity for people with advanced cancer, and the positivist-empiricist definitions of physical activity and exercise. ${ }^{24}$ Participants engaged in activity to meet their minimum level of responsibility, rather than to improve health or fitness. This is congruent with the extant literature: people with advanced cancer reported a functional, rather than reflexive, account of the individual self: "the emphasis was what might be described as 'functional' - on 'doing' and 'being' - rather than a cognitive process of maintaining meaning" (p.183). ${ }^{32}$

The emergent theoretical framework identified activity and day-to-day routine as potential mechanisms for participants to meet their minimum level of responsibility. This is similar to Ruijs et al's (2013) findings that impairment in routine daily activities was more prevalent, and contributed to more distress in people with advanced cancer, than impaired leisure activities. ${ }^{33}$ La Cour et al (2009) explored how advanced cancer patients fashioned a meta-narrative of "saying goodbye in a good way", within which the emplotment of routine activity provided reassurance that life continues in the midst of incurable illness. Morgan et al. (2015) showed that active participation in everyday activities was one means by which advanced cancer patients mediated adjustment to functional deterioriation at the end of life. ${ }^{34}$ Taken together, the meaning of activity for people with advanced cancer is less associated with improving health or fitness, and more so to functional, day-to-day behaviours which enable them to fulfil tasks or duties. 


\section{Perceptions of Activity}

The second objective of this thesis was to elicit people with advanced cancer's perceptions of activity with respect to their quality of life. Participants did not perceive activity as the universal means of addressing their main concern, due to the dynamic interplay of perceived benefits and prevailing conditions in influencing downsizing to the critical threshold. In the face of uncertainty in disease progression and symptom burden, the person with advanced cancer continues on with whatever they are able to manage ${ }^{35}$. Under conditions of high day-to-day fluctuations and low physical functioning, mechanisms involving sedentary behaviour (i.e. pacing and path of least resistance) take precedence for the person with advanced cancer to meet their critical threshold of responsibility.

The grounded theory demonstrates that at any given moment, the person with advanced cancer scales back to the minimum level of responsibility possible in order to conserve energy. Johnston et al. (2012) report that limiting activities was the most common way that people with advanced cancer in the last year of life managed 'overwhelming tiredness' ${ }^{36}$. Reeve et al. (2010) describe the 'felt exhaustion' of people with advanced cancer preceding periods of biographical upset, during which they have insufficient energy levels to complete daily activities; there is a need to restore depleted energy in order to sustain the continuity of the daily routine ${ }^{32}$.

The findings of this study draw parallels to the perceptions of activity in non-cancer populations. In a qualitative study of African Americans with Class III/IV heart failure, the theme of "given up" emerged from participant narratives of current physical activity. Despite an 
expressed desire to be physically active, one person with advanced stage cardiac failure reported "'I don't really do nothing now. I just do things for myself, just for me...I never lost interest. But I just can't do it no more'" 37. Participant narratives of their typical day showed very little activity, which was attributed to the low level of physical functioning and conditioning consistent with advanced stage cardiac failure. The grounded theory explains that, under the condition of low physical functioning, people with advanced cancer likewise prioritise their most essential responsibilities at that point in time, and do no more than that.

\section{Barriers and Facilitators to Activity}

The third objective of this thesis was to elicit people with advanced cancer's views of barriers and facilitators to activity in the context of their day-to-day life. In this study, the ability of participants to engage in activity varied with the changing conditions within the emergent theoretical framework. The prevailing conditions of understanding of illness, symptom burden, acute stressors and person supports can impede or facilitate activity on a day-to-day basis.

Parallels can be drawn with the experience of activity in non-cancer populations. In a qualitative study exploring physical activity in community-dwelling people aged 80-91 years old, being more active and improving physical fitness was not the primary motivator; participants described the meaning of physical activity as embedded in everyday activities, and purposefully restricted physical activity due to fear of falling and inducing pain and fatigue. ${ }^{38}$ In a qualitative study of African Americans with Class III/IV heart failure, participant narratives of their typical day showed very little activity, which was attributed to the low level of physical functioning and conditioning consistent with advanced stage cardiac failure. ${ }^{37}$ Reeve et al. (2010) describe how 
advanced cancer imposes an embodied cost, in the form of fatigue and energy depletion, to individual efforts to maintain continuity of daily routine. Cancer is described as having an embodied, rather than cognitive, effect: as one person with advanced cancer reported, " 'It

drains you...I feel with my cancer, it's hard work.' " 32. Perceived as a physical adversary, disease burden can be a barrier to activity in people with advanced cancer.

Implications

The grounded theory explains the discrepancy between expressed interest and actual participation in a physical activity intervention from previous research in people with advanced cancer ${ }^{10-12}$. Expressed interest in physical activity was a confirmation of shared positive assumptions about physical activity, rather than a reflection of the underlying motivations and day-to-day experience of people with advanced cancer. The assumptions with which the researcher approaches the research problem and method, may impact study outcomes ${ }^{39}$; the predominantly positivist-empiricist assumptions underlying physical activity research in different populations need to be examined.

Activity is important to people with advanced cancer, but not through the positivist-empiricist lens which is used predominantly in the extant literature. Participants view activity as the dayto-day behaviour which enables them to meet their critical threshold of responsibility, rather than as a means of improving health and fitness. Any level of physical exertion may be all that the person with advanced cancer is able to undertake. As such, testing exercise interventions using objective fitness measures are not appropriate in people with advanced cancer and a life expectancy of less than four months. Future research should explore how one can modify 
barriers and enhance facilitators to everyday activity in this population, using concepts that emerged from, and nomenclature that is aligned with, the advanced cancer experience.

In a discussion of physical activity research in persons with disabilities, Wheeler (1998) argues that "we must consider the context in which we collect our data, what our work means to the subjects, and the value judgements that are routinely made in the questions that researchers ask and the methods they use to find the answers" ${ }^{40}$. In this study, qualitative inquiry was guided by exploration of participants' perceptions of activity, alongside their day-to-day behaviour. Both positivist and interpretivist approaches, and quantitative and qualitative methodologies, are clearly warranted in future research in activity and advanced cancer.

The grounded theory emerged from the advanced cancer experience, and the meaning of activity is unique for people with advanced cancer. The language and constructs of activity which clinicians use may not be congruent with how activity is experienced by people with advanced cancer. The issues of greatest importance to people with advanced cancer may not be the same for interdisciplinary team members who are involved in their clinical care. Clinicians should focus on supporting, and being aware of the barriers and facilitators to, everyday activity of people with advanced cancer.

\section{Evaluating Grounded Theory}

The criterion of fit questions whether the concept adequately expresses the pattern in the data which it intends to conceptualize. ${ }^{27}$ Through the iterative use of constant comparative analysis, this theory bears witness to the criterion of fit; the core conceptual category could not have been anticipated a priori, and the resulting theory was not constructed to match the initial 
study aim and objectives. The criterion of work questions whether the concepts and proposed conceptual relationships sufficiently explain the behaviour in a substantive area and account for how the main concern of participants is resolved. ${ }^{27}$ Close adherence to a classic grounded theory approach, including the abeyance of the literature review, has ensured that the emergent concepts stayed true to the latent pattern of behaviour of the participants under study and were not based on preconceptions. The criterion of relevance questions whether the theory being conceptually grounded in the data is reflective of the significance of the participants' main concern. ${ }^{27}$ The analytic questions used to interrogate the data, ensured the relevance of this theory to the participants' main concern; the core conceptual category likewise resonates with clinical experience working with people with advanced cancer. The criterion of modifiability is specific to the propositional nature of classic grounded theory, and questions the openness of theory to being modified as new data is constantly compared to generate new categories, properties and dimensions. ${ }^{27}$ This theory bears witness to modifiability in that constant comparative analysis yielded new sub-categories, and further articulated properties and dimensions, which were integrated into the final theory.

\section{Strengths and Limitations}

This is an original substantive theory that increases understanding of the experience of day-today behaviour of people with advanced cancer. To our knowledge, this is the first study to utilize an accelerometer as a qualitative probe in people with advanced cancer. As all participants were recruited from an outpatient palliative care department at a tertiary cancer centre in Alberta, Canada, the scope of the grounded theory is limited to the chosen site and population. In order to increase its scope to that of a middle range theory, substantive 
formalization is required and would entail sampling different substantive groups, contexts, and social units. ${ }^{14}$ Future research should examine people with advanced cancer within different care settings, across Canada, or in different countries, as data for constant comparative analysis for the grounded theory.

\section{Conclusion}

Meeting their minimum level of responsibility was the primary explanatory driver underlying the day-to-day behaviour of people with advanced cancer. People with advanced cancer experienced activity as the day-to-day behaviours which enabled them to fulfil the obligations that they deem most important. People with advanced cancer perceived activity as one possible means of meeting their minimum level of responsibility. Their ability to engage in activity, however, was limited by varying conditions at any given moment in time. Symptom burden, day-to-day fluctuations and acute stressors modified the Critical Threshold and were barriers to activity. Expressed interest, therefore, was not matched by the ability to engage in activity by people with advanced cancer. Through adherence to classic grounded theory methodology, this substantive theory emerged that is aligned with the advanced cancer experience. The grounded theory should inform research and clinical practice by focussing on the issues of greatest importance to the individual, and which may ultimately impact quality of life for people with advanced cancer.

\section{Acknowledgements}

The authors would like to thank the people with advanced cancer, their caregivers and families for the gift of their time and participation. 


\section{Authors' Note}

This study was conducted in partial fulfilment of the first author's PhD in Palliative Care degree at Lancaster University, under the supervision of SGB (80\%) and CM (20\%).

\section{Declaration of conflicting interests}

The author(s) declared no potential conflicts of interest with respect to the research, authorship and/or publication of this article.

\section{Funding}

The author(s) disclosed receipt of the following financial support for the research, authorship and/or publication of this article: Alberta Cancer Foundation Roche Fellowship Award in Translational Research (first author).

\section{References}

1. Crum RM, Anthony JC, Bassett SS and Folstein MF. Population-based norms for the Mini-Mental State Examination by age and educational level. JAMA : the journal of the American Medical Association. 1993; 269: 2386-91.

2. Anderson F, Downing GM, Hill J, Casorso L and Lerch N. Palliative performance scale (PPS): a new tool. J Palliat Care. 1996; 12: 5-11.

3. NCCN. National Comprehensive Cancer Network (NCCN) Clinical practice guidelines in oncology: cancer-related fatigue. 2016.

4. Jordhoy MS, Inger Ringdal G, Helbostad JL, Oldervoll L, Loge JH and Kaasa S. Assessing physical functioning: a systematic review of quality of life measures developed for use in palliative care. Palliat Med. 2007; 21: 673-82.

5. Fong DYT, Ho JWC, Hui BPH, et al. Physical activity for cancer survivors: meta-analysis of randomised controlled trials. Br Med J. 2012; 344: 14.

6. Mishra SI, Scherer RW, Snyder C, Geigle PM, Berlanstein DR and Topaloglu O. Exercise interventions on health-related quality of life for people with cancer during active treatment. The Cochrane database of systematic reviews. 2012; 8: Cd008465. 
7. Speck RM, Courneya KS, Masse LC, Duval S and Schmitz KH. An update of controlled physical activity trials in cancer survivors: a systematic review and meta-analysis. Journal of cancer survivorship : research and practice. 2010; 4: 87-100.

8. Giesinger JM, Wintner LM, Oberguggenberger AS, et al. Quality of life trajectory in patients with advanced cancer during the last year of life. Journal of palliative medicine. 2011; 14: 904-12.

9. Heywood R, McCarthy AL and Skinner TL. Safety and feasibility of exercise interventions in patients with advanced cancer: a systematic review. Supportive care in cancer : official journal of the Multinational Association of Supportive Care in Cancer. 2017; 25: 3031-50.

10. Lowe SS, Watanabe SM, Baracos VE and Courneya KS. Physical activity interests and preferences in palliative cancer patients. Supportive care in cancer : official journal of the Multinational Association of Supportive Care in Cancer. 2010; 18: 1469-75.

11. Lowe SS, Watanabe SM, Baracos VE and Courneya KS. Associations Between Physical Activity and Quality of Life in Cancer Patients Receiving Palliative Care: A Pilot Survey. J Pain Symptom Manage. 2009; 38: 785-96.

12. Lowe SS, Watanabe SM, Baracos VE and Courneya KS. Home-based functional walking program for advanced cancer patients receiving palliative care: a case series. BMC Palliat Care. 2013; 12: 9.

13. Glaser and Strauss. The discovery of grounded theory: strategies for qualitative research. New York: Aldine de Gruyter, 1967.

14. Holton JA and Walsh I. Classic Grounded Theory: Applications with Qualitative \& Quantitative Data. Los Angeles: SAGE, 2017.

15. Madill A. Realism. In: Given LM, (ed.). The SAGE Encyclopedia of Qualitative Research Methods. Thousand Oaks: Sage, 2008, p. 732-6.

16. Maxwell JA. A realist approach for qualitative research. Thousand Oaks: Sage, 2012.

17. Lowe SS, Milligan C, Watanabe SM and Brearley SG. A Grounded Theory Approach to Physical Activity and Advanced Cancer: A Qualitative Study Protocol. Int J Qual Meth. 2015; 14: 10.

18. Glaser. Doing Grounded Theory: issues and discussions. Mill Valley: Sociology Press, 1998.

19. Lowe SS, Tan M, Faily J, Watanabe SM and Courneya KS. Physical activity in advanced cancer patients: a systematic review protocol. Systematic reviews. 2016; 5: 43.

20. Lowe SS, Watanabe SM and Courneya KS. Physical activity as a supportive care intervention in palliative cancer patients: a systematic review. The journal of supportive oncology. 2009; 7: 27-34.

21. Fairchild A, Pituskin E, Rose B, et al. The rapid access palliative radiotherapy program: blueprint for initiation of a one-stop multidisciplinary bone metastases clinic. Supportive care in cancer : official journal of the Multinational Association of Supportive Care in Cancer. 2009; 17: 163-70.

22. Glaser. Theoretical sensitivity. Mill Valley, CA: Sociology Press, 1978.

23. Payne S. Qualitative methods of data collection and analysis. In: Addington-Hall JM, Bruera E, Higginson IJ and Payne S, (eds.). Research Methods in Palliative Care. Oxford: Oxford University Press, 2007, p. 139-61.

24. Bouchard C and Shephard RJ. Physical activity, fitness and health: the model and key concepts. In: Bouchard C, Shephard RJ and Stephens T, (eds.). Physical activity, fitness and health - International proceedings and consensus statement. Champaign, IL: Human Kinetics, 1994, p. 77-88.

25. Friese S. Computer-Assisted Grounded Theory Analysis. In: Equit C and Hohage C, (eds.). Handbuch Grounded Theory - Von der Methodologie zur Forschungspraxis. Weinheim: Beltz Juventa, 2016, p. 483-507.

26. Engward H. Understanding grounded theory. Nursing standard (Royal College of Nursing (Great Britain) : 1987). 2013; 28: 37-41.

27. Glaser. Basics of grounded theory analysis: emergence vs. forcing. Mill Valley, CA: Sociology Press, 1992. 
28. Gulde I, Oldervoll LM and Martin C. Palliative Cancer Patients' Experience of Physical Activity. J Palliative Care. 2011; 27: 296-302.

29. Malcolm L, Mein G, Jones A, Talbot-Rice H, Maddocks M and Bristowe K. Strength in numbers: patient experiences of group exercise within hospice palliative care. BMC Palliat Care. 2016; 15: 8.

30. Turner K, Tookman A, Bristowe K and Maddocks M. 'I am actually doing something to keep well. That feels really good': Experiences of exercise within hospice care. Prog Palliat Care. 2016; 24: 204-12.

31. Downing $M$, Lau $F$, Lesperance $M$, et al. Meta-analysis of survival prediction with Palliative Performance Scale. J Palliat Care. 2007; 23: 245-52; discussion 52-4.

32. Reeve J, Lloyd-Williams M, Payne $S$ and Dowrick C. Revisiting biographical disruption: exploring individual embodied illness experience in people with terminal cancer. Health: An Interdisciplinary Journal for the Social Study of Health, Illness \& Medicine. 2010; 14: 178-95 18p.

33. Ruijs CD, Kerkhof AJ, van der Wal G and Onwuteaka-Philipsen BD. Symptoms, unbearability and the nature of suffering in terminal cancer patients dying at home: a prospective primary care study. $B M C$ family practice. 2013; 14: 201.

34. Morgan DD, Currow DC, Denehy L and Aranda SA. Living actively in the face of impending death: constantly adjusting to bodily decline at the end-of-life. BMJ supportive \& palliative care. 2015.

35. McKechnie R, MacLeod R and Keeling S. Facing uncertainty: the lived experience of palliative care. Palliat Support Care. 2007; 5: 367-76.

36. Johnston B, Milligan S, Foster C and Kearney N. Self-care and end of life care-patients' and carers' experience a qualitative study utilising serial triangulated interviews. Support Care Cancer. 2012; 20: 1619-27.

37. McCarthy M, Katz SD, Schipper J and Dickson VV. "I Just Can't Do It Anymore" Patterns of Physical Activity and Cardiac Rehabilitation in African Americans with Heart Failure: A Mixed Method Study. Healthcare (Basel, Switzerland). 2015; 3: 973-86.

38. Welmer AK, Morck A and Dahlin-Lvanoff S. Physical Activity in People Age 80 Years and Older as a Means of Counteracting Disability, Balanced in Relation to Frailty. J Aging Phys Act. 2012; 20: 317-31.

39. Craig P, Dieppe P, Macintyre S, Michie S, Nazareth I and Petticrew M. Developing and evaluating complex interventions: the new Medical Research Council guidance. BMJ (Clinical research ed). 2008; 337: a1655.

40. Wheeler GD. Challenging our assumptions in the biological area of adapted physical activity: A reaction to Shephard (1998). Adapt Phys Act Q. 1998; 15: 236-49. 


\section{Appendix A: Semi-structured Interview Guide}

- The semi-structured interview will be guided by the activPAL ${ }^{\mathrm{TM}}$ activity monitor output and daily record sheets that the participant will have completed over the previous week

- The activPAL ${ }^{\mathrm{TM}}$ activity monitor output and daily record sheets will be different for each participant, and will therefore act as a unique interview probe for each participant

- The activPAL ${ }^{\mathrm{TM}}$ activity monitor output will be printed out and shared with the participant at the beginning of the interview, with subsequent interview questions informed by the patterns therein as well as the participant's reactions and observations

- Sample Questions (may vary depending on participant's activity monitor output and daily record sheets)

- Please describe to me what happened on this day...

○ What else was happening that day when your activity was...

- Are you surprised by what you see on the activity monitor output. Why or why not?

- Why do you think your activity pattern is the way it is?

- Would you like to engage in that activity more or less? Why or why not?

- Are these usual daily activities important to you? If so, why?

- Did your usual daily activities change with having cancer? If so, how?

- What do your usual daily activities mean to you?

- What would hinder you from engaging in your usual daily activities?

- What helps you to engage in your usual daily activities?

- What does quality of life mean to you?

- Do you think your quality of life and your usual daily activities are related? If so, how? 